\title{
Batch Fabrication of Micro-Coils for MR Spectroscopy on Silicon
}

\author{
R.R.A. Syms, M.M.Ahmad, I.R.Young, D.Gilderdale \\ EEE Dept, \\ Imperial college \\ Exhibition Road, London SW7 2AZ, UK \\ Email:r.syms@ic.ac.uk
}

\begin{abstract}
A process for batch fabrication of low-cost microcoils for magnetic resonance (MR) spectroscopy of clinical biopsy samples is demonstrated. Conductors are fabricated on oxidized, intrinsic silicon substrates by electroplating metals inside a deep photoresist mould. Through-wafer deep reactive ion etching (DRIE) is used to define precise sample volumes and stencil cuts around each die, and dies are separated by snap-out. Single- and multiple-coil (Helmholtz-type) sensors are formed, by stacking parts on silicon baseplates. MR imaging and spectroscopy are both demonstrated on model samples, with high SNR.
\end{abstract}

\section{INTRODUCTION}

There is increasing evidence that magnetic resonance spectroscopy (MRS) may be useful in evaluating cancerous lesions [1], and MR spectra have now been obtained from a variety of body organs including the brain $[2,3]$. When a brain tumor is resected, a large number $(>12)$ of biopsy samples may be taken. Samples obtained with a biopsy needle are often cylindrically shaped, and around $1 \mathrm{~mm}$ in diameter and 5-10 mm long. The samples are used to determine the cause and form of the tumor and to locate its boundaries. The components of interest in MRS (which is almost invariably based on ${ }^{1} \mathrm{H}$ spectroscopy) exist in tissue in 0.5 to $5 \mathrm{mmol}$ quantities. To obtain data quickly, close coupling is needed between the sample and the MR detector. Small coils, which are expensive and require careful handling, are therefore used. One major potential problem is sterilization between patients. Ideally, the coils should therefore be both cheap and disposable.

In MRS, the signal-to-noise ratio should be maximized, so that the advantages obtained from close coupling to the signal are not lost [4]. Coil resistance and capacitive leakage must be minimized, so a high Q-factor may be obtained from a resonant detector. The most common operating frequency in clinical MRI is $63.8 \mathrm{MHz}$ - the resonance frequency of protons in a $1.5 \mathrm{~T}$ magnetic field. At this frequency, the skin depth $\delta=1 / \sqrt{ }\left(\pi f \mu_{0} / \rho\right)$ is $\approx 8.3 \mu \mathrm{m}$ for $\mathrm{Cu}$. Conductors thickness up to $2 \delta=16 \mu \mathrm{m}$ are therefore used to minimize resistance. Methods of combining thick conductors with substrates that can be etched to provide sampling troughs are therefore desirable.

\author{
D.J.Collins, M.O.Leach \\ Royal Marsden NHS Trust \\ Downs Road, Sutton, Surrey SM2 5PT, UK
}

Planar MR detectors were originally fabricated by photolithographic patterning by Peck et al. [5, 6]. More recently, coils have been combined with sample volumes on glass by Dechow et al. [7], implantable devices have been developed by Renaud et al. [8], and integrated preamplifiers have been developed [9]. The highest Q-factors are obtained on insulating substrates such as glass [10]. However, modest performance can be obtained on oxidized intrinsic silicon using MEMS technologies. For example, deep reactive ion etching (DRIE) is a method of near vertical etching that operates by a cyclic repetition of etching and passivation processes [11]. DRIE has a high rate ( $>2 \mu \mathrm{m} / \mathrm{min})$, and may etch through a $500 \mu \mathrm{m}$ thick wafer in under 4 hours. In addition to forming sample volumes, DRIE may also be used to segment a wafer, thus eliminating the dicing step, which is often dirty, and time-consuming.

In this paper, we show that deep etching of silicon may be used to combine structuring steps that segment the wafer and define sample locations with electroplating steps that form the coil. The fabrication process is described in Section II. Electrical characterization and magnetic resonance imaging and spectroscopy are described in Section III, and conclusions are reached in Section IV.

\section{DESIGN AND FABRICATION}

\section{A. Design}

Micro-coils are formed as shown in Figure 1a. Here a wafer is first patterned to define an electroplated conductor. Single-turn conductors may be formed using single-layer patterning, and multi-turn conductors using multi-layer lithography. The wafer is then patterned and etched to define a locating trough for the sample and a stencil cut that passes around the coil, apart from short sections of connecting sprue. When the sprue is broken, the coil is released from the wafer without the need for dicing or major cleaning. Components such as base-plates carrying additional circuitry, as shown in Figure 1b, may also be fabricated. Figure 1c shows how single coils may be mounted on a base-plate to form a sample trough with a closed base. Figure 1d shows how parts may be stacked to form a Helmholtz coil. One coil component is flip-chip bonded to a baseplate, while a second is stacked on top of the first and wire-bonded to complete the coil winding. 


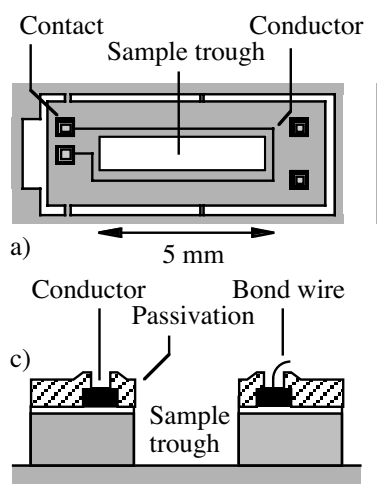

Baseplate

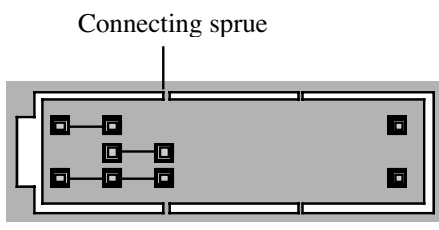

b)

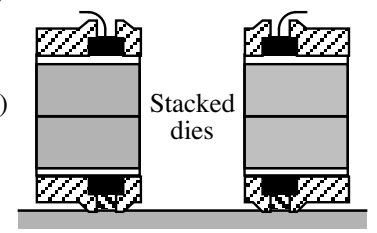

Solder connection to tracking
Figure 1. a) Micro coil; b) baseplate; c) coil assembly; d) Helmholtz coil assembly.

\section{B. Fabrication}

Figure 2 shows the fabrication process, which is based entirely on non-magnetic materials. A $100 \mathrm{~mm}$ diameter $<100>$ oriented, intrinsic $(>5000 \Omega \mathrm{cm}$ ) Si wafer is first thermally oxidized to form an isolation layer (step 1). We have used $1.5 \mu \mathrm{m}$ of $\mathrm{SiO}_{2}$. The oxide is then coated with an adhesion layer of $300 \AA \mathrm{Ti}$ and a seed layer of $2000 \AA \mathrm{Cu}$ by RF sputtering (step 2). A thick (22 $\mu \mathrm{m})$ layer of AZ6290 photoresist (Clariant UK Ltd.) is then spin coated over the wafer, and patterned by UV lithography using the conductor mask to form a deep mould. the conductors are formed by depositing a $14 \mu \mathrm{m}$ thick $\mathrm{Cu}$ layer using an acid plating solution (FB, from Technic Inc.) and a $4 \mu \mathrm{m}$ thick Au layer using a thiosulphate/sulphite solution (ECF 60, from Metalor) (step 3).
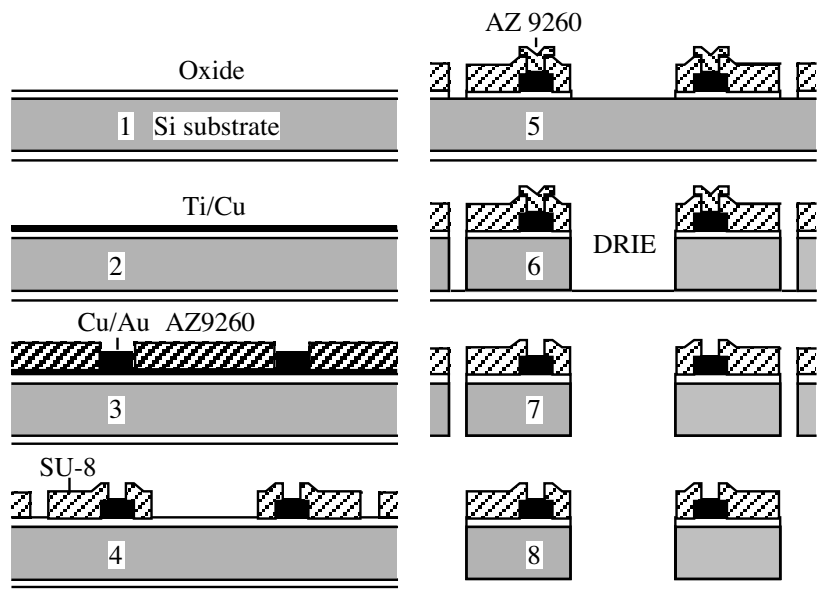

Figure 2. Fabrication of micro-coils by deep etching of silicon.

The mould is then removed by a combination of wet and dry stripping. A $20 \mu \mathrm{m}$ thick layer of SU-8 2050 (Chestech, UK), a rugged, epoxy-based resist [12], is then deposited by spin coating. This layer forms a mask for deep reactive ion etching and also protects the coil during subsequent use (step 4). The contact pads are protected with a layer of AZ9260 resist during etching. The $\mathrm{SiO}_{2}$ is etched using a Plasma Technology System $80^{+}$parallel plate reactive ion etcher, using $\mathrm{CHF}_{3}, \mathrm{O}_{2}$ and $\mathrm{Ar}$ gases (step 5). The wafer is then etched using a Surface Technology Systems Single Chamber Multiplex inductively coupled plasma etcher, operating a variant of the cyclic Bosch/STS advanced silicon etch [11]. The wafer is etched right through, at a rate of $2 \mu \mathrm{m} / \mathrm{min}$ (step 6). Dual-frequency plasma is used to combine high-rate etching with stop-on-oxide. Residual oxide is then removed from the back-side of the wafer by reactive ion etching in the System $80^{+}$. The resist pads are then removed from the contacts using a combination of wet and dry stripping.

Prototype devices were fabricated as single-tirn coils with $100 \mu \mathrm{m}$ wide conductors and $1 \mathrm{~mm} \times 5 \mathrm{~mm}$ sample troughs. The die size was $3 \mathrm{~mm} \times 11 \mathrm{~mm}$. Figure 3 a shows part of a completed wafer carrying both coil and base-plate dies. Snap-out die separation was successfully demonstrated with high yield. Single coils were then mounted on small PCBs, and pairs of coils were stacked to form Helmholtz coils. In the latter case, the dies were aligned mechanically, and epoxied together. Electrical connections were formed by bump bonding with using indium solder. Figure $3 \mathrm{~b}$ shows (left to right) i) a coil, ii) a coil mounted on a small PCB, iii) a base-plate, iv) a coil attached to a baseplate, v) a Helmholtz coil, and vi) a Helmholtz coil mounted on a PCB.

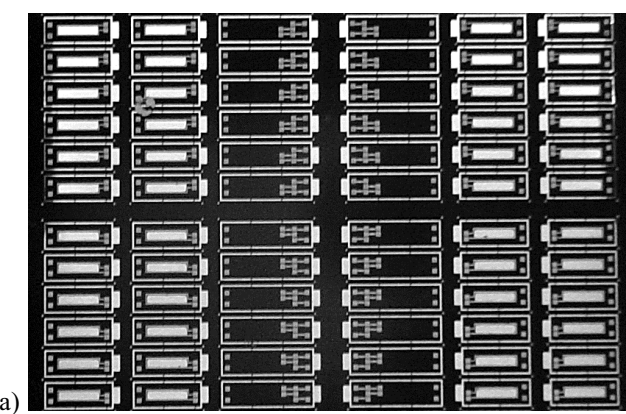

b)

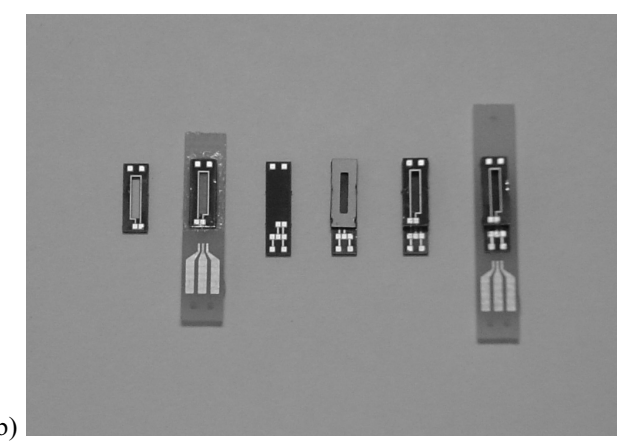

Figure 3. a) Dies before snap-out and b) coil components during assembly

\section{Evaluation}

\section{A. Electrical measurements}

Q-factors of isolated and resonant coils were measured with an Agilent E5061A network analyzer. Single coils had an inductance of $9 \mathrm{nH}$, which rose to $11 \mathrm{nH}$ after mounting on a silicon base-plate. Helmholtz coils had an inductance of $24 \mathrm{nH}$ after wire-bonding to a small PCB. Coil assemblies 
were configured as parallel resonators using non-magnetic surface mount capacitors. High Q-factors were obtained, showing that good performance can still be achieved despite the use of a silicon substrate and soldered interconnects.

Figure $4 \mathrm{a}$ shows the frequency variation of the impedance of resonant detectors based on a single-turn coil, configured for operation at $63.8 \mathrm{MHz}$ (i.e., in a $1.5 \mathrm{~T}$ field) and $127.6 \mathrm{MHz}(3 \mathrm{~T})$. In the former case, Q-factor is 15 and the shunt impedance at resonance is $60 \Omega$. In the latter case, the Q-factor has risen to 22 . Figure $4 \mathrm{~b}$ shows the frequency variation of the $\mathrm{Q}$-factor, which peaks at $\approx 25$ at around 200 $\mathrm{MHz}$, suggesting that good performance would still be obtained in a $6 \mathrm{~T}$ field. Resonant Helmholtz detectors had a Q-factor of 13 when configured for operation at $63.8 \mathrm{MHz}$.

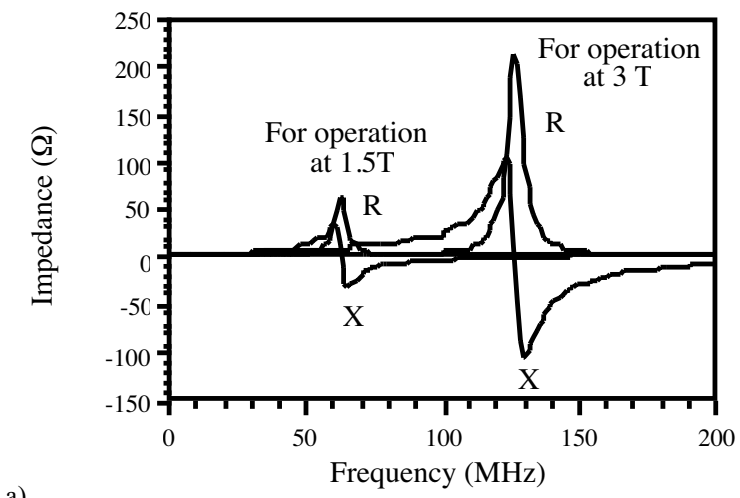

a)

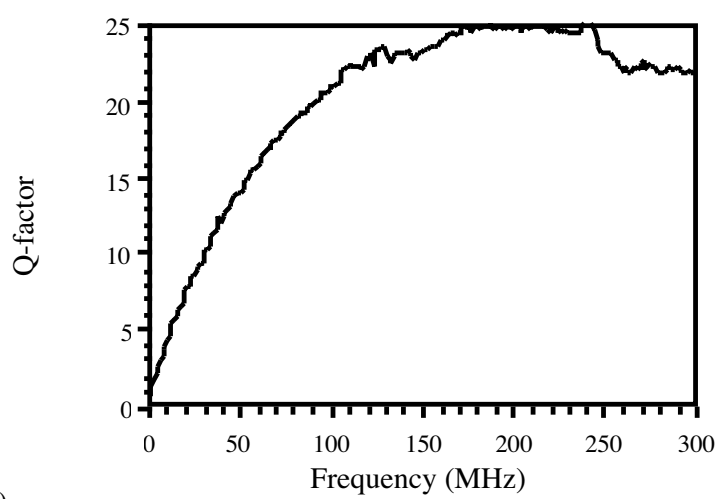

Figure 4. Frequency variation of a) the impedance and b) the Q-factorof single turn resonators.

\section{B. MR experiments}

Magnetic resonance experiments were performed using a 1.5 T Siemens Magnetom Vision MRI system at the Royal Marsden Hospital. The main body coil was used for RF transmission, and the microcoils were used for signal reception. Additional PIN diode-switched detuning circuitry was provided to protect the microcoil during transmission, as shown in Figure 5. Here the capacitor $C_{T}$ is used for tuning, the capacitor $C_{M}$ for matching to $50 \Omega$ and the inductor $\mathrm{L}_{\mathrm{D}}$ for detuning.

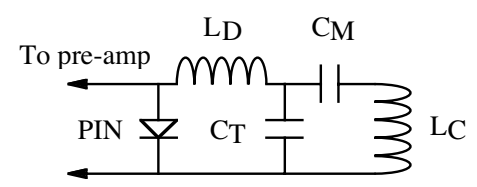

Figure 5. Circuit for tuning, matching and protection.

Microcoils are sensitive to both interior and exterior signal sources, and interior imaging was first demonstrated. The sample was a slice of a "Spenco" dermal pad, a vinyl plastisol material from Spenco Healthcare International, Horsham, West Sussex, UK which has previously been used as an internal signal source in fiducial marker coils [13].

Figure 6a shows a coronal slice image of a Spenco sample taken from a single-turn coil. The slice is a horizontal cut through a sliver of Spenco measuring $3 \times 4.5$ x $0.7 \mathrm{~mm}$, placed upright in the sample trough. The images were obtained using a 28 × $28 \mathrm{~mm}$ field of view, a $500 \mu \mathrm{m}$ slice thickness and four signal averages from a $35 \mathrm{~ms}$ spin echo sequence. Excellent SNR is clearly obtained. Figure $6 \mathrm{~b}$ shows a similar image obtained from a Helmholtz coil; here the image definition is rather better.

Imaging of an exterior sample was then demonstrated, using a semi-cylindrical cod-liver oil capsule placed directly on top of a single turn coil. The capsule diameter was $\approx 8$ $\mathrm{mm}$. Figure $6 \mathrm{c}$ shows a transverse slice image. The majority of the capsule is in view, but the signal clearly reduces with distance from the coil plane, falling to around $10 \%$ of its peak at $\approx 2.4 \mathrm{~mm}$ above the coil. This image was obtained using a $56 \times 56 \mathrm{~mm}$ field of view, a $500 \mu \mathrm{m}$ slice thickness and two signal averages of a $35 \mathrm{~ms}$ spin echo sequence.

a)

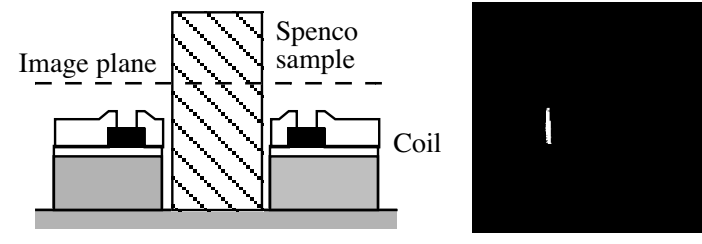

b)
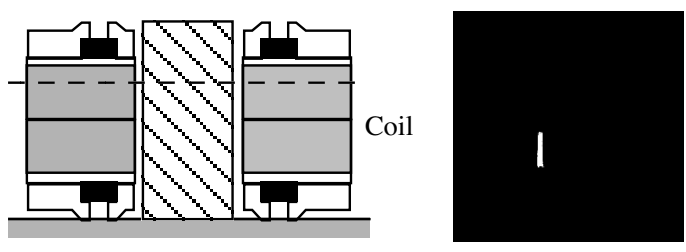

c)

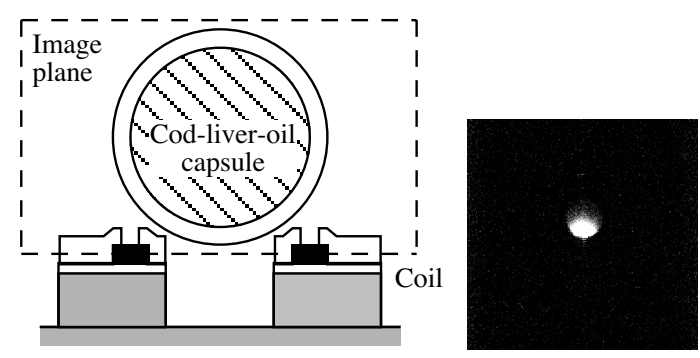

Figure 6. MR imaging of a), b) Spenco and c) cod liver oil. 
Magnetic resonance spectroscopy of both types of sample was then demonstrated. Figure 7 a shows a MR spectrum obtained from the Spenco sample using a single turn coil. The spectrum consists of a large, narrow peak with two clearly resolved side peaks, which is characteristic of this material. The measured line-width of the main peak was $54 \mathrm{~Hz}$.

Figure $7 \mathrm{~b}$ shows the spectrum of the cod-liver oil sample. The line-width of the lipid peak is $12-17 \mathrm{~Hz}$. The signal to noise ratio was estimated from the peak signal divided by the RMS value of the noise in the absence of signal as $\sim 900$. The data shown have been apodized to boost high frequencies; this procedure helps to identify doublets, which can otherwise appear as a single broad peak. Considerable structure may be seen in the spectrum, and assignment of the peaks is in progress.

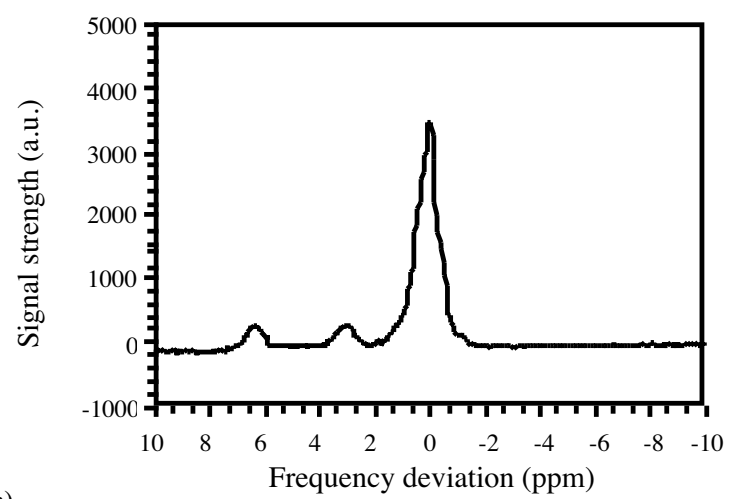

a)

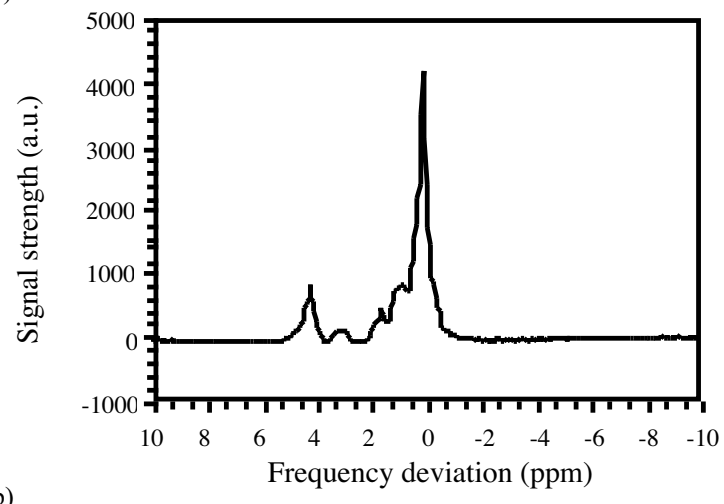

Figure 7. MR spectra obtained from a) Spenco and b) cod-liver oil.

\section{CONCLUSIONS}

Deep reactive ion etching and electroplating have been combined in a wafer-scale process for batch fabrication of magnetic resonance detectors on oxidised silicon substrates. The conductors are formed first, by electroplating metals inside a photoresist mould, and the die topography is then defined by deep reactive ion etching. Detectors may be detached from the wafer by a simple snap-out process and assembled into a variety of configurations, including single turn and Helmholtz coils.

Q-factors up to 15 have been obtained at $63.8 \mathrm{MHz}$, despite the use of a semiconducting substrate. Preliminary MRI and MRS experiments have been performed, and excellent SNR and line-width have been obtained. Further improvements in signal obtained from optimised multi-turn coil geometries and from an increase in magnetic field strength are expected. An obvious near-term target is to perform similar evaluations of tissue samples, and this work is in progress. Alternative needle coil geometries are now being investigated, to allow tissue samples to be assessed without the need to remove them from a biopsy needle.

\section{ACKNOWLEDGMENT}

The Authors are grateful to Dr J. Stagg for DRIE work.

\section{REFERENCES}

[1] C.E. Mountford, S. Doran, C.L. Lean, P. Russell, "Cancer pathology in the year 2000" Biophys. Chem., vol 68, pp. 127-135, 1997.

[2] S.J. Barton, F.A. Howe, A.M. Tomlins, S.A. Cudlip, J.K. Nicholson, B.A. Bell, J.R. Griffiths, "Comparison of in vivo ${ }^{1} \mathrm{H}$ MRS of human brain tumours with ${ }^{1} \mathrm{H}$ HR-MAS spectroscopy of intact biopsy samples in vitro" Magma, vol 8, pp. 121-8, 1999.

[3] H. Poptani, J. Kaartinen, R.K. Gupta, M. Niemitz, Y. Hiltunen, R.A. Kauppinen, "Diagnostic assessment of brain tumours and nonneoplastic brain disorders in vivo using proton nuclear magnetic resonance spectroscopy and artificial neural networks" J. Cancer Res. Clin. Oncol., vol 125, pp. 343-9, 1999.

[4] D.J. Gilderdale, N.M. deSouza, G.A. Coutts, M.K. Chui, D.J. Larkman, A.D. Williams, "Design and use of internal receiver coils for magnetic resonance imaging" Brit. J. Radiol., vol 72, pp. 1141-51, 1999.

[5] T.L. Peck, R.L. Magin, J. Kruse, M. Feng, "NMR microspectroscopy on 100- $\mu \mathrm{m}$ planar RF coils fabricated on gallium arsenide substrates" IEEE T. Biomed. Eng., vol 41, pp. 706-9, 1994.

[6] T.L. Peck, R.L. Magin, P.C. Lauterbur, "Design and analysis of microcoils for NMR microscopy", J. Magn. Reson. Ser. vol. B108, pp. 114-124, 1995

[7] J. Dechow, A. Forchel, T. Lanz, A. Haase "Fabrication of NMRmicrosensors for nanoliter sample volumes" Microelectr. Engng., vol. 53, pp. 517-519, 2000.

[8] L. Berry, L. Renaud, P.Kleimann, P. Morin, M. Armenean, H. SaintJalmes, "Development of implantable detection microcoils for minimally invasive NMR spectroscopy" Sensors and Actuators vol. A93, pp. 214-218, 2001.

[9] J. Dechow, T.Lanz, M. Stumber, A.Forchel, A. Haase, "Preamplified planar microcoil on GaAs substrates for microspectroscopy", Rev. Sci. Instrum. vol. 74, pp. 4855-4857, 2003.

[10] C. Massin, C. Boero, F. Vincent, J. Abenhaim, P.A. Besse, R.S. Popovic, "High-Q factor RF planar microcoils for micro-scale NMR spectroscopy” Sensors and Actuators vol. A97-98, pp. 280-288, 2002.

[11] J.K. Bhardwaj, H. Ashraf, "Advanced silicon etching using high density plasmas", Proc. SPIE vol. 2639, pp. 224-233, 1995.

[12] E.H. Conradie, D.F. Moore, "SU-8 thick photoresist processing as a functional material for MEMS applications", J. Micromech.Microeng. vol. 12, pp. 368-374, 2002.

[13] G.A. Coutts, D.J. Gilderdale, K.M. Chui, L. Kasuboski, N.M. deSouza, "Integrated and interactive position tracking and imaging of interventional and internal devices using small fiducial receiver coils" Magn. Reson. Med. vol. 40, pp. 908-913, 1998. 